\title{
A Unified Integro-Differential Nonlocal Model
}

\author{
Parisa Khodabakhshi ${ }^{\mathrm{a}}$, J.N. Reddy, ${ }^{\mathrm{b}, *}$ \\ ${ }^{a}$ Department of Civil Engineering, Texas AESM University \\ ${ }^{b}$ Department of Mechanical Engineering, Texas AEM University \\ College Station, TX77843-3123
}

${ }^{*}$ Please address correspondence to Professor J.N. Reddy, e-mail: jnreddy@t amu . edu 


\begin{abstract}
In this paper a unified integro-differential nonlocal elasticity model is presented and its use in the bending analysis of Euler-Bernoulli beams is illustrated. A general (for an elastic continuum) finite element formulation for the two-phase integro-differential form of Eringen nonlocal model is provided. The equations are specialized for the case of the Euler-Bernoulli beam theory. Several numerical examples, including the paradoxical cantilever beam problem that eluded other researchers, are provided to show how the present nonlocal model affects the transverse displacement of beams. The examples show that Eringen nonlocal constitutive relation has a softening effect on the beam, except for the case of the simply supported beam. A brief discussion on the applicability of the integrodifferential model to other problems is also presented. Finally, the transition from the stiffened nonlocal simply supported beam to the softened nonlocal clamped beam is also investigated.
\end{abstract}

Keywords: Eringen nonlocal model, Integro-differential formulation, Euler-Bernoulli beam, Cantilever beam

\title{
1. Introduction
}

For hyperelastic materials (i.e., Green elastic materials) there exists a potential function whose derivative with respect to the strain at a point gives the corresponding stress at that point (Reddy, 2013). This forms the basis for local (conventional) constitutive model where the stress and strain at each point are related. Local theory of continuum mechanics is inherently scale free, i.e. forces are only transferred through contact and no long-range forces between points located further apart is considered. However, there exists certain phenomena (e.g., dispersion of elastic waves, crack propagation in fracture mechanics, dislocations, and so on) that cannot be explained using local theory of elasticity. In addition, as a consequence of recent developments in the filed of material science there is a need to model the structural response of a variety of new materials that require the consideration of nonlocal aspects of the material (e.g. size effect in nanomaterials). In nonlocal theories, stress at each point is influenced by the strain at all points in the domain. This influence decreases as the distance between the points increases. The concept of nonlocal theory of linear elasticity was initially introduced in papers by Kröner (1967), Krumhansl (1968), and Kunin (1968). Later, 
the idea of long-range interactions was further developed in the works of Eringen (1972b,a, 1983, 2002) and Eringen and Edelen (1972). Eringen (1983) introduced an integro-differential nonlocal model which has widely been used in the literature. Later, Eringen proposed a two-phase nonlocal model (Eringen, 1987) which was a combination of local and integro-differential nonlocal constitutive theory. One of the advantages of an integral nonlocal theory over the local elasticity theory is that the former gives non-singular results for geometric singularities (i.e. cracks) due to the averaging effect inherent in the integral form of the constitutive relation.

The nonlocal integral constitutive equation makes use of a positive distancedecaying kernel function which specifies the dependence of stress at each point on the strain at other points in the domain. Eringen (1983) showed that for a specific class of kernel functions the Eringen nonlocal integral constitutive equation can be transformed into a differential form with the exact same properties. Due to the difficulties in using integral constitutive equations, the nonlocal differential model proposed by Eringen (1983) is the one most widely used in the literature to account for nonlocal effects. Several studies have been reported on the basis of nonlocal theories. Peddieson et al. (2003) used the Eringen nonlocal differential model to derive the equations of equilibrium for a nonlocal Euler-Bernoulli beam. This study (Peddieson et al., 2003) was pioneering in the sense that Eringen nonlocal differential model was used to incorporate nonlocal effects into the analysis of structural elements. One of the main issues that was discussed in the work of Peddieson et al. (2003) was the fact that in nonlocal cantilever beams (enhanced with Eringen's differential model) nonlocal effects were not triggered for point loads applied at the free end. This is not a desirable outcome, because recently cantilever beams of micro- and nano- size have found several applications as actuators and sensors in the field of chemical and biological sciences (Pereira, 2001; J. Pei and Thundat, 2004; Ekinci and Roukes, 2005; N. V. Lavrik and Datskos, 2004). If a nonlocal model is not capable of capturing the size effect in these nano- and macro- cantilever beams, then the data obtained by these devices may not be interpreted correctly.

Other examples of nonlocal Euler-Bernoulli beam studies were presented in Sudak (2003); Challamel and Wang (2008); Lu et al. (2006); Shakouri et al. (2009). Challamel and Wang (2008) also pointed to the deficiency mentioned in Peddieson et al. (2003) and suggested the integration of gradient elasticity model with Eringen nonlocal model to eliminate it. Shakouri et al. (2009) gave a discrete formulation for nonlocal Euler-Bernoulli beam representation of the doublewalled carbon nanotubes using the Galerkin method. Wang et al. (2008, 2006), 
Wang and Wang (2007), and Wang and Liew (2007) integrated Timoshenko beam theory with Eringen nonlocal model. The main problem with these works (Wang et al., 2008; Wang and Wang, 2007; Wang and Liew, 2007; Wang et al., 2006) is that nonlocal effects are only limited to normal stresses and not transverse shear stresses. Reddy (2007) used Eringen nonlocal model to give the variational statements for several beam theories, namely the Euler-Bernoulli, Timoshenko, Reddy and Levinson beam theories. In this comprehensive study (Reddy, 2007) the above-mentioned limitation imposed in the works of Wang et al. (2008, 2006); Wang and Liew (2007); Wang and Wang (2007) is removed and nonlocal effects are included in both normal and transverse shear stresses. Analytical solutions of static bending, vibration, and buckling of the beams are also provided in this study. Later, Reddy (2010) formulated the governing equations for the bending of beams (Euler-Bernoulli and Timoshenko beam theories) and plates (Classical and first order shear deformation plate theories) which also took in account von Kármán nonlinearity. Reddy (2010) stated that no quadratic functional can be derived for the differential form of Eringen nonlocal beam theory from which the governing equations can be derived. Thai (2012), and Thai and Vo (2012) recently provided a higher order nonlocal beam theory which is slightly different from Reddy beam theory which also accounted for variation of shear stress along the height of the beam. Reddy and El-Borgi (2014) provided the governing equations for bending of nonlocal Euler-Bernoulli and Timoshenko beam theories accounting for moderate rotations through modified von Kármán nonlinearity. Several studies have also applied Eringen nonlocal model to the study of functionally graded beams (Reddy et al., 2014; Rahmani and Pedram, 2014; Salehipour et al., 2015). Studies on nonlocal beam theories based on the differential model are far more exhaustive to be reported here. Interested readers may consult Reddy and El-Borgi (2014) and Reddy et al. (2014).

In all of the above-mentioned references, the differential form of the Eringen model had been used. Polizzotto (2001) applied the integral form of Eringen model and derived the variational principles governing the integral form from which the nonlocal finite element formulation is obtained. The kernel function in the integral constitutive equation brings in a concept of the length scale. Pisano and Fuschi (2003) used the approach proposed by Polizzotto (2001) to derive a closed-form solution for a bar in tension with nonlocal Eringen model as the constitutive equation. Later, Pisano et al. (2009) used this integro-dfferential nonlocal model to give a finite element formulation for 2D problems of two-phase elastic materials (Eringen, 1987). Di Paola et al. (2011) came up with a new method to introduce long-range forces into the equations of motion. General 3D variational 
statements were constructed and they were further simplified for the Timoshenko beam theory. The formulation proposed by Di Paola et al. (2011) is conceptually similar to the formulation of peridynamic theory proposed by Silling (2000).

It is found by several authors that Eringen's differential model yields inconsistent results for a cantilever when compared to other boundary conditions (Peddieson et al., 2003; Challamel and Wang, 2008; Wang and Liew, 2007; Wang et al., 2008; Challamel et al., 2014). For all boundary conditions except the cantilever, the model predicts softening effect (i.e., larger defections and lower fundamental frequencies) as the nonlocal parameter is increased. Several ad hoc approaches or explanations have been proposed to alleviate the baffling case of the cantilever beam. In the present study, classical theory of elasticity is augmented with Eringen's nonlocal model in integral form to present a unified integro-differential model for nonlocal elasticity and a general finite element formulation for the integral form of Eringen nonlocal model. Note that by using the two-phase Eringen model (Eringen, 1987), two control parameters will exist, namely the length scale parameter and phase parameter. The general 3D equations are further simplified to the one-dimensional case of the Euler-Bernoulli beam theory. Several examples are provided to show how Eringen nonlocal model affects the transverse displacement of the beams. In this study, the kernel function used in the integral constitutive equation is different from that of which yields into Eringen's differential equation (Eringen, 1983). It is shown that the proposed nonlocal model yields consistent results for most boundary conditions (including the paradoxical case of a cantilever beam), however, the results are slightly different for the case of a simply supported beam. Among the provided examples, other than the simply supported beam which shows a slight stiffening effect, the rest of the boundary conditions show a softening effect as expected. This result is promising, in the sense that it can be used for the study of nonlocal effects in micro- and nanocantilevers used as actuators/sensors in biological and chemical sciences. The softening effect increases with the increase in the length scale parameter and the decrease in the phase parameter. A brief discussion on the applicability of the integral formulation to general problems is brought in the end. Also the transition from stiffened nonlocal simply supported beam to softened nonlocal clamped beam is studied further. 


\section{Eringen-type Nonlocal Formulation}

\subsection{Unified Nonlocal Constitutive Model}

The Eringen nonlocal model (Eringen, 1972b,a) is based on the assumption that the stress at each point depends on the strain at all points of the domain. According to this model, stress at point $\mathbf{x}$ can be determined according to the following equation:

$$
\boldsymbol{\sigma}(\mathbf{x})=\int_{V^{\prime}} \alpha\left(\mathbf{x}, \mathbf{x}^{\prime}, l_{c}\right) \mathbf{C}: \boldsymbol{\varepsilon}\left(\mathbf{x}^{\prime}\right) d V^{\prime}
$$

where, $\boldsymbol{\varepsilon}\left(\mathbf{x}^{\prime}\right)$ is the local strain at point $\mathbf{x}^{\prime}$ which is assumed to be the linearized version of the Green-Lagrange strain tensor (Reddy, 2013):

$$
\boldsymbol{\varepsilon}(\mathbf{x})=\frac{1}{2}\left[\nabla \mathbf{u}+(\nabla \mathbf{u})^{T}\right]
$$

In equation $1, \alpha\left(\mathbf{x}, \mathbf{x}^{\prime}, l_{c}\right)$ is a kernel function which determines the measure by which stress at point $\mathbf{x}$ is affected by the strain at point $\mathbf{x}^{\prime} ; \alpha\left(\mathbf{x}, \mathbf{x}^{\prime}, l_{c}\right)$ has the following properties (Eringen, 1983):

1. It should be a positive distance decaying function which its maximum value takes place at $\mathbf{x}=\mathbf{x}^{\prime}$.

2. In the limit of $l_{c} \rightarrow 0$ (where $l_{c}$ is a length scale parameter), the kernel function should revert to the Dirac-delta function and the nonlocal formulation should simplify to the familiar local formulation.

3. In the limit of $l_{c} \rightarrow \infty$, the Eringen model should approximate lattice theory.

4. The integral of this function over the whole domain should be unity (assuming that point $\mathbf{x}$ is embedded in an infinite domain):

$$
\int_{V^{\prime}} \alpha\left(\mathbf{x}, \mathbf{x}^{\prime}, l_{c}\right) d V^{\prime}=1
$$

Selection of the kernel function is based on satisfying the above-mentioned physical and mathematical requirements. Note that the kernel function introduces a concept of material length scale into the problem. The distance of each point within the domain from a specific point $\mathbf{x}$ becomes meaningful only when it is compared to a length scale. Kernel function is often chosen to be symmetric and 
one of the most commonly used forms is the exponential function of the following form:

$$
\alpha\left(\frac{\left|\mathbf{x}-\mathbf{x}^{\prime}\right|}{l_{c}}\right)=\alpha_{0} e^{-\frac{\left|\mathbf{x}-\mathbf{-}^{\prime}\right|}{l_{c}}}
$$

where $\alpha_{0}$ is determined by satisfying the constraint in equation 3 while assuming that the point is embedded in an infinite domain.

Equation 1 can be modified so that it takes a form of a two-phase constitutive model with both local and nonlocal phases (Eringen, 1987):

$$
\boldsymbol{\sigma}(\mathbf{x})=\xi_{1} \mathbf{C}: \boldsymbol{\varepsilon}(\mathbf{x})+\int_{V^{\prime}} \xi_{2} \alpha\left(\mathbf{x}, \mathbf{x}^{\prime}, l_{c}\right) \mathbf{C}: \boldsymbol{\varepsilon}\left(\mathbf{x}^{\prime}\right) d V^{\prime}
$$

where $\xi_{1}$ and $\xi_{2}$ satisfy the following relation:

$$
\xi_{1}+\xi_{2}=1
$$

Here $\xi_{1}$ and $\xi_{2}$ represent measures of local and nonlocal properties of the model; $\xi_{1}=1$ and $\xi_{2}=0$ corresponds to the purely local constitutive equation and $\xi_{2}=1$ and $\xi_{1}=0$ corresponds to the original Eringen nonlocal constitutive equation; $\xi_{1}$ is called phase parameter in the following sections.

It is worth mentioning that nonlocal theories in general will introduce at least one length scale parameter into the constitutive equation (i.e. $l_{c}$ in equation 1 ) regardless of the choice of differential or integral form. The two-phase constitutive equation used in this study (5) accounts for an additional independent variable, i.e. the phase parameter, compared to the differential form of Eringen nonlocal model. The phase parameter, $\xi_{1}$ adds to the generality of the formulation used herein. By setting $\xi_{1}=0$ original Eringen formulation is attained.

In order for the nonlocal continuum theory to be applicable to the study of problems with prominent nonlocal effects, the length scale parameter, $l_{c}$, should be determined to be representative of the problem at hand. Notice that in the original formulation of Eringen (1983) and the papers using the differential form of Eringen model, the kernel function is defined to be $\alpha=\alpha\left(\left|\mathbf{x}-\mathbf{x}^{\prime}\right|, \tau\right)$, where $\tau=e_{0} a / l$. In this formulation $a$ and $l$ are internal and external characteristic lengths, respectively, and $e_{0}$ is a constant which is material dependent. The corresponding counterpart in the formulation used herein is $\tau l=e_{0} a=l_{c}$. To this date, no consensus has been reached on how to experimentally determine the material-dependent length scale parameter. Eringen (1983) proposed that the length scale parameter be determined such that the dispersion curve of the nonlocal theory agreed with that of the atomistic approaches. Wang and Wang (2007) suggested a conservative 
range for the length scale parameter of the single-walled CNTs to be $l_{c}<2.0 \mathrm{~nm}$. According to Arash and Wang (2012), the length scale parameter of CNTs depends on several parameters, i.e. boundary conditions, chirality, number of walls, and so on. Further studies are required to determine the length scale parameter corresponding to a specific problem.

\subsection{General Finite Element Formulation}

The equations of equilibrium and the boundary conditions will remain the same as local analysis, where $\sigma$ is replaced by the definition in equation 5:

$$
\mathbf{u}=\overline{\mathbf{u}} \quad \begin{array}{ccc}
\nabla . \boldsymbol{\sigma}+\mathbf{b}=\mathbf{0} & \text { in } V \\
\text { on } \Gamma_{u}, & \mathbf{t}=\overline{\mathbf{t}} \quad \text { on } \Gamma_{t}
\end{array}
$$

where, $V$ is the interior domain of the body, $\Gamma_{u}$ is the part of the boundary where displacements are specified, and $\Gamma_{t}$ is the part of the boundary where tractions are specified; $\Gamma_{u}$ and $\Gamma_{t}$ are disjoint parts with the property $\Gamma_{u} \cup \Gamma_{t}=\Gamma$, with $\Gamma$ being the entire boundary of the domain.

The total potential energy of the system can be written as (Reddy, 2002):

$$
\begin{aligned}
\Pi(\mathbf{u})= & U+V^{E}=\int_{V}\left(\frac{1}{2} \boldsymbol{\sigma}: \boldsymbol{\varepsilon}-\mathbf{b} \cdot \mathbf{u}\right) d V-\oint_{\Gamma} \mathbf{t} . \mathbf{u} d S \\
= & \int_{V}\left(\frac{1}{2} \xi_{1} \boldsymbol{\varepsilon}(\mathbf{x}): \mathbf{C}: \boldsymbol{\varepsilon}(\mathbf{x})-\mathbf{b} . \mathbf{u}\right) d V \\
& +\frac{1}{2} \int_{V}\left(\int_{V^{\prime}} \xi_{2} \alpha\left(\mathbf{x}, \mathbf{x}^{\prime}, l_{c}\right) \boldsymbol{\varepsilon}(\mathbf{x}): \mathbf{C}: \boldsymbol{\varepsilon}\left(\mathbf{x}^{\prime}\right) d V^{\prime}\right) d V-\oint_{\Gamma} \mathbf{t} . \mathbf{u} d S
\end{aligned}
$$

The finite element model is derived using the procedure discussed in (Reddy, 2006). The weak form associated with equation 8 is

$$
\begin{aligned}
0= & \int_{V_{e}}\left\{-(\nabla \mathbf{w})^{T}:\left[\xi_{1} \mathbf{C}: \boldsymbol{\varepsilon}_{e}(\mathbf{x})+\sum_{e^{\prime}=1}^{N_{e l}} \int_{V_{e^{\prime}}} \xi_{2} \alpha\left(\mathbf{x}, \mathbf{x}^{\prime}, l_{c}\right) \mathbf{C}: \boldsymbol{\varepsilon}_{e^{\prime}}\left(\mathbf{x}^{\prime}\right) d V^{\prime}\right]\right\} d V \\
& +\int_{V_{e}} \mathbf{w}^{T} \cdot \mathbf{b}_{e} d V+\oint_{\Gamma_{e}} \mathbf{w}^{T} \cdot \mathbf{t}_{e} d S
\end{aligned}
$$

where, $N_{e l}$ is the number of elements in the domain and $\mathbf{t}_{e}$ is the traction vector along the boundaries of element $e$. The nonlocal terms correspond to element $e^{\prime}$ which interacts with element $e$. 
Next, the displacement field $\mathbf{u}(\mathbf{x})$ is replaced with a finite element approximation and the strains are computed using equation 2 :

$$
\begin{gathered}
\mathbf{u}(\mathbf{x})=\Psi_{e} \boldsymbol{\Delta}_{e} \quad \mathbf{x} \in V_{e}, e=1, \ldots, N_{e l} \\
\boldsymbol{\varepsilon}(\mathbf{x})=\left\langle\begin{array}{llllll}
\varepsilon_{x x} & \varepsilon_{y y} & \varepsilon_{z z} & \gamma_{y z} & \gamma_{x z} & \gamma_{x y}
\end{array}\right\rangle^{T}=\mathbf{D u}(\mathbf{x})=\mathbf{B}_{e} \boldsymbol{\Delta}_{e}
\end{gathered}
$$

In equations 10 and $11, \Delta_{e}$ is the vector of nodal displacements of element $e$, and $\Psi_{e}$ is the matrix of shape functions relating the displacement field of the element to its nodal displacements $\Delta_{e}$. For a general three-dimensional problem $\Delta_{e}$, and $\Psi_{e}$ are defined according to equations 12 and 13 with $n$ being the number of nodes in the element.

$$
\begin{aligned}
\boldsymbol{\Delta}_{e} & =\left\langle\begin{array}{ccccccc}
u_{x}^{1} & u_{y}^{1} & u_{z}^{1} & \ldots & u_{x}^{n} & u_{y}^{n} & u_{z}^{n}
\end{array}\right\rangle_{3 n \times 1}^{T} \\
\boldsymbol{\Psi}_{e} & =\left[\begin{array}{ccccccc}
\psi_{1} & 0 & 0 & \ldots & \psi_{n} & 0 & 0 \\
0 & \psi_{1} & 0 & \ldots & 0 & \psi_{n} & 0 \\
0 & 0 & \psi_{1} & \ldots & 0 & 0 & \psi_{n}
\end{array}\right]_{3 \times 3 n}
\end{aligned}
$$

In equation $11, \mathbf{B}_{e}$ is

$$
\mathbf{B}_{e}=\mathbf{D} \Psi_{e}
$$

and $\mathbf{D}$ is a differential matrix operator:

$$
\mathbf{D}=\left[\begin{array}{ccc}
\frac{\partial}{\partial x} & 0 & 0 \\
0 & \frac{\partial}{\partial y} & 0 \\
0 & 0 & \frac{\partial}{\partial z} \\
0 & \frac{\partial}{\partial z} & \frac{\partial}{\partial y} \\
\frac{\partial}{\partial z} & 0 & \frac{\partial}{\partial x} \\
\frac{\partial}{\partial y} & \frac{\partial}{\partial x} & 0
\end{array}\right]
$$

The weight function $\mathbf{w}$ is replaced with the shape functions $\Psi_{e}$. Therefore, we have

$$
\nabla \mathbf{w}=\mathbf{B}_{e}=\mathbf{D \Psi}_{e}
$$

Finally, $\mathbf{u}$ and $\mathbf{w}$ are substituted into equation 9 to obtain the final finite element model over an element $e$

$$
\begin{aligned}
0= & \int_{V_{e}}\left\{-\mathbf{B}_{e}^{T}:\left[\xi_{1} \mathbf{C}: \mathbf{B}_{e} \boldsymbol{\Delta}_{e}+\sum_{e^{\prime}=1}^{N_{e l}} \int_{V_{e^{\prime}}} \xi_{2} \alpha\left(\mathbf{x}, \mathbf{x}^{\prime}, l_{c}\right) \mathbf{C}: \mathbf{B}_{e^{\prime}} \boldsymbol{\Delta}_{e^{\prime}} d V^{\prime}\right]+\Psi_{e}^{T} \mathbf{b}_{e}\right\} d V \\
& +\oint_{\Gamma_{e}} \Psi_{e}^{T} \mathbf{t}_{e} d S
\end{aligned}
$$


or

$$
\xi_{1} \mathbf{k}_{e}^{l} \boldsymbol{\Delta}_{e}+\xi_{2} \sum_{e^{\prime}=1}^{N_{e l}} \mathbf{k}_{e e^{\prime}}^{n l} \boldsymbol{\Delta}_{e^{\prime}}=\mathbf{F}_{e}
$$

where

$$
\begin{aligned}
\mathbf{k}_{e}^{l} & =\int_{V_{e}} \mathbf{B}_{e}^{T}: \mathbf{C}: \mathbf{B}_{e} d V \quad \mathbf{x} \in V_{e} \\
\mathbf{k}_{e e^{\prime}}^{n l} & =\int_{V_{e}} \int_{V_{e^{\prime}}} \alpha\left(\mathbf{x}, \mathbf{x}^{\prime}, l_{c}\right) \mathbf{B}_{e}^{T}: \mathbf{C}: \mathbf{B}_{e^{\prime}} d V^{\prime} d V \quad \mathbf{x} \in V_{e}, \mathbf{x}^{\prime} \in V_{e}^{\prime} \\
\mathbf{F}_{e} & =\int_{V_{e}} \Psi_{e}^{T} \mathbf{b}^{e} d V+\oint_{\Gamma_{e}} \Psi_{e}^{T} \mathbf{t}^{e} d S
\end{aligned}
$$

where $\mathbf{k}_{e}^{l}$ and $\mathbf{k}_{e e^{\prime}}^{n l}$ are the local and nonlocal parts of the stiffness matrix, respectively; $\mathbf{F}_{e}$ is the element force vector. Note that $\mathbf{k}_{e e^{\prime}}^{n l}$ has double integration over the domain of element $e$ and $e^{\prime}$. By setting $\xi_{1}=1$ in equation (18), we obtain the familiar finite element model for the local theory. Also it is worth mentioning that according to equation (21) the natural boundary conditions for nonlocal formulation remains the same as those of local formulation. Equation (19) is assembled for all elements in the domain and the specified boundary conditions are imposed before solving for the unknown nodal values of the displacements.

\subsection{Kernel Function for One-dimensional Analysis}

This paper is dedicated to the study of beams with nonlocal constitutive equations. Therefore, one needs to determine the one dimensional kernel function used in Eringen nonlocal model. It is assumed that nonlocal effects are only present along the length of the beam and no effect is considered in the other two dimensions. For 1D problems equation (4) simplifies to an exponential equation of the following form:

$$
\alpha\left(\frac{\left|x-x^{\prime}\right|}{l_{c}}\right)=\alpha_{0} e^{-\frac{\left|x-x^{\prime}\right|}{l_{c}}}
$$

The constraint in equation (3) is used to determine the constant $\alpha_{0}$ in equation (22). For simplicity of integration, it is assumed that the cross section of the beam is constant and doesn't change across the length of the beam, therefore, $A^{\prime}=A$ :

$$
\int_{-\infty}^{+\infty} \int_{A} \alpha\left(\frac{\left|x-x^{\prime}\right|}{l_{c}}\right) d A d x^{\prime}=2 \alpha_{0} A l_{c}=1 \rightarrow \alpha_{0}=\frac{1}{2 A l_{c}}
$$


Hence the kernel function becomes

$$
\alpha\left(\frac{\left|x-x^{\prime}\right|}{l_{c}}\right)=\frac{1}{2 A l_{c}} e^{-\frac{\left|x-x^{\prime}\right|}{l_{c}}}
$$

However, note that the nonlocal part of the stiffness matrix (equation (20)) has a volume integral over the domain of element $e^{\prime}$. Knowing that no nonlocal effects is considered along the height and width of the beam, $A$ can be factored out of the integration. Finally, one can assume the kernel function to be:

$$
\alpha\left(\frac{\left|x-x^{\prime}\right|}{l_{c}}\right)=\frac{1}{2 l_{c}} e^{-\frac{\left|x-x^{\prime}\right|}{l_{c}}}
$$

where, the volume integration over element $e^{\prime}$ is transformed to an integration over the length of this element only.

In the original formulation, each point is assumed to interact nonlocally with all other points in the domain. Since the kernel function, $\alpha$, decays rapidly with distance, it acquires a very small value beyond some point. One can make use of this property to limit the domain of nonlocal interactions which is named to be the influence zone. An influence zone of the radius $l_{i}=6 l_{c}$ is a reasonable choice which is used in this study. Using this value the exponential term of the kernel function reduces to $e^{-l_{i} / l_{c}} \approx 0.0025$ which is a practical small value.

\subsection{Finite Element Model of the Nonlocal Euler-Bernoulli Beam Theory}

The ideas presented in the previous sections are applied to the bending of beams. We consider the Euler-Bernoulli beam theory to illustrate the application of the integro-differential model proposed herein. We also show the cantilever beam paradox can be successfully resolved.

Consider a straight, homogeneous, isotropic beam of length $L$, constant rectangular cross section $b \times h$ ( $b$ being the width and $h$ being the height). The $x$-axis is taken along the geometric centroid of the beam cross section and the $\mathrm{z}$-coordinate is taken positive upward. The only nonzero strain in the Euler Bernoulli beam theory (Reddy, 2006) is

$$
\varepsilon(x)=\frac{d u}{d x}-z \frac{d^{2} w}{d x^{2}}
$$

The nonlocal stress is determined using equation (5):

$$
\sigma(x)=\xi_{1} E\left(\frac{d u}{d x}-z \frac{d^{2} w}{d x^{2}}\right)+\int_{0}^{L} \int_{A^{\prime}} \xi_{2} \alpha\left(x, x^{\prime}, l_{c}\right) E\left(\frac{d u}{d x}-z \frac{d^{2} w}{d x^{2}}\right) d A^{\prime} d x^{\prime}
$$


Applying the general calculations of section (2.2) to the present case, one can derive the one-dimensional finite element formulation for an element of a beam under the action of distributed horizontal and transverse loads, $f(x)$ and $q(x)$ , respectively. Note that, since the governing equation for the Euler-Bernoulli beam is a fourth order equation, integration by parts is applied twice for the term containing $w$. This makes the calculations slightly different from those of section (2.2). The finite element formulation takes the form of equation (18):

$$
\xi_{1}\left[\begin{array}{cc}
\left(\mathbf{k}_{e}^{l}\right)^{11} & \mathbf{0} \\
\mathbf{0} & \left(\mathbf{k}_{e}^{l}\right)^{22}
\end{array}\right]\left\{\begin{array}{c}
\boldsymbol{\Delta}_{e}^{1} \\
\boldsymbol{\Delta}_{e}^{2}
\end{array}\right\}++\xi_{2} \sum_{e^{\prime}=1}^{N_{e l}}\left[\begin{array}{cc}
\left(\mathbf{k}_{e e^{\prime}}^{n l}\right)^{11} & \mathbf{0} \\
\mathbf{0} & \left(\mathbf{k}_{e e^{\prime}}^{n l}\right)^{22}
\end{array}\right]\left\{\begin{array}{c}
\boldsymbol{\Delta}_{e^{\prime}}^{1} \\
\boldsymbol{\Delta}_{e^{\prime}}^{2}
\end{array}\right\}=\left\{\begin{array}{l}
\mathbf{F}_{e}^{1} \\
\mathbf{F}_{e}^{2}
\end{array}\right\}
$$

where,

$$
\begin{gathered}
\left(\mathbf{k}_{e}^{l}\right)_{i j}^{11}=\int_{x_{1}^{e}}^{x_{2}^{e}} E A^{e} \frac{d \psi_{i}^{e}}{d x} \frac{d \psi_{j}^{e}}{d x} d x, \quad i, j=1 . .2 \\
\left(\mathbf{k}_{e}^{l}\right)_{i j}^{22}=\int_{x_{1}^{e}}^{x_{2}^{e}} E A^{e} \frac{d^{2} \phi_{i}^{e}}{d x^{2}} \frac{d^{2} \phi_{j}^{e}}{d x^{2}} d x, \quad i, j=1 . .4 \\
\left(\mathbf{k}_{e e^{\prime}}^{n l}\right)_{i j}^{11}=\int_{x_{1}^{e}}^{x_{2}^{e}} \int_{x_{1}^{e^{\prime}}}^{x_{2}^{e^{\prime}}} \alpha\left(x, x^{\prime}, l_{c}\right) E A^{e} A^{e^{\prime}} \frac{d \psi_{i}^{e}}{d x} \frac{d \psi_{j}^{e^{\prime}}}{d x} d x^{\prime} d x, \quad i, j=1 . .2 \\
\left(\mathbf{k}_{e e^{\prime}}^{n l}\right)_{i j}^{22}=\int_{x_{1}^{e}}^{x_{2}^{e}} \int_{x_{1}^{e^{\prime}}}^{x_{2}^{e^{\prime}}} \alpha\left(x, x^{\prime}, l_{c}\right) E I^{e} A^{e^{\prime}} \frac{d^{2} \phi_{i}^{e}}{d x^{2}} \frac{d^{2} \phi_{j}^{e^{\prime}}}{d x^{2}} d x^{\prime} d x, \quad i, j=1 . .4 \\
\left(\Delta^{e}\right)^{1}=\left\langle u_{1}^{e} \quad u_{2}^{e}\right\rangle^{T}, \quad\left(\Delta^{e}\right)^{2}=\left\langle w_{1}^{e} \theta_{1}^{e} w_{2}^{e} \quad \theta_{2}^{e}\right\rangle^{T} \quad\left(\theta_{i}^{e}=-\left.\frac{d w}{d x}\right|_{x=x_{i}^{e}}\right) \\
\left(\mathbf{F}_{e}\right)_{i}^{1}=\int_{x_{1}^{e}}^{x_{2}^{e}} f(x) \psi_{i}^{e} d x+\sum_{j=1}^{2} P_{j} \psi_{j}^{e}\left(x_{i}^{e}\right), \quad i, j=1 . .2 \\
\left(\mathbf{F}_{e}\right)_{i}^{2}=\int_{x_{1}^{e}}^{x_{2}^{e}} q(x) \phi_{i}^{e} d x+\sum_{j=1}^{4} Q_{j} \phi_{j}^{e}\left(x_{i}^{e}\right), \quad i, j=1 . .4
\end{gathered}
$$

and $\psi_{i}^{e}$ and $\phi_{i}^{e}$ are the linear Lagrange and Hermite cubic interpolations functions, respectively. Since the cross section of the beam is assumed to be constant, the subscripts $e$ and $e^{\prime}$ over $A$ and $I$ in equations (29) and (30) can be eliminated. Also in the above equations $x_{1}^{e}\left(x_{1}^{e^{\prime}}\right)$, and $x_{2}^{e}\left(x_{2}^{e^{\prime}}\right)$ are the $x$-coordinate of the first and last 
node of element $e\left(e^{\prime}\right)$, respectively. Substituting for $\alpha\left(x, x^{\prime}, l_{c}\right)$ from equation (24) one can rewrite $\mathbf{k}_{e e^{\prime}}^{n l}$ as

$$
\begin{aligned}
& \left(\mathbf{k}_{e e^{\prime}}^{n l}\right)_{i j}^{11}=\int_{x_{1}^{e}}^{x_{2}^{e}} \int_{x_{1}^{e^{\prime}}}^{x_{2}^{e^{\prime}}} \frac{1}{2 l_{c}} e^{-\frac{\left|x-x^{\prime}\right|}{l_{c}}} E A^{e} \frac{d \psi_{i}^{e}}{d x} \frac{d \psi_{j}^{e^{\prime}}}{d x} d x^{\prime} d x, \quad i, j=1 . .2 \\
& \left(\mathbf{k}_{e e^{\prime}}^{n l}\right)_{i j}^{22}=\int_{x_{1}^{e}}^{x_{2}^{e}} \int_{x_{1}^{e^{\prime}}}^{x_{2}^{e^{\prime}}} \frac{1}{2 l_{c}} e^{-\frac{\left|x-x^{\prime}\right|}{l_{c}}} E I^{e} \frac{d^{2} \phi_{i}^{e}}{d x^{2}} \frac{d^{2} \phi_{j}^{e^{\prime}}}{d x^{2}} d x^{\prime} d x, \quad i, j=1 . .4
\end{aligned}
$$

The final assembled form of the equations is

$$
\left(\xi_{1} \mathbf{K}^{l}+\xi_{2} \mathbf{K}^{n l}\right) \mathbf{U}=\mathbf{F}
$$

where $\mathbf{U}$ and $\mathbf{F}$ are the global vectors of nodal displacements and nodal forces, respectively, and $\mathbf{K}^{l}$ and $\mathbf{K}^{n l}$ are the assembled local and nonlocal global stiffness matrices, respectively.

\section{Numerical Results}

\subsection{General Comments}

In this section, numerical results are presented for the Euler-Bernoulli beam theory with nonlocal constitutive relation of the form in equation 27 . Four different types of boundary conditions and loads are considered (Figure 1) here:

(a) Clamped beam with uniformly distributed load

(b) Simply supported beam with uniformly distributed load

(c) Cantilever beam with a concentrated load applied to the free end of the beam

(d) Cantilever beam with non-uniform triangularly distributed load

In all cases, a uniform rectangular cross section with width $b$ and height $h$ is considered. The problems are solved for three different values of length scale parameter, $l_{c}$, and five different values of phase parameter, $\xi_{1}$. The value of the length scale parameter is chosen to be much smaller than the length of the beam. The nonlocal interactions are considered to exist only within the influence zone. If the mesh is such that only part of an element falls into this zone, the nonlocal interaction between that element and the element of interest is neglected. This causes the discretized integral form of equation 18 to be mesh-dependent to some 


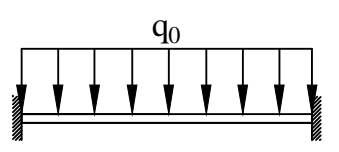

(a)

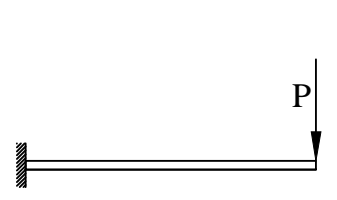

(c)

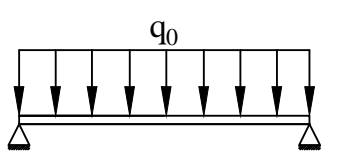

(b)

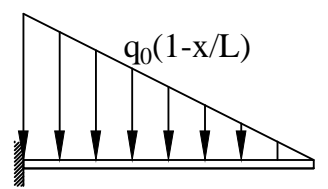

(d)

Figure 1: Analysis cases with different load conditions and boundary conditions.

degree for coarse meshes which will be discussed later. The integral equations are solved numerically using Gauss-Legendre quadrature rule.

In all of the examples presented here the length to height ratio of the beam is chosen to be 40 . Therefore, assumption of a thin beam theory is appropriate and the fact that Euler-Bernoulli beam theory neglects shear deformation does not introduce a significant error into the solution.

\subsection{Clamped Beam with Uniform Distributed Load}

The first example deals with a clamped beam of length $L$, constant bending stiffness $E I$, and uniformly distributed load of intensity $q_{0}$. The maximum deflection according to the local beam model is $w(L / 2)=q_{0} l^{4} / 384 E I$. For a beam with constant $E I$, the conventional FEM gives the exact deflection. The nonlocal deflecitons are normalized with respect to the maximum deflection of the conventional beam. The deflections of the beam for the nonlocal case $\left(\xi_{1}=0\right)$ and 3 different values of $l_{c}$, namely $l_{c}=0.01 \mathrm{~L}, 0.015 \mathrm{~L}$, and $0.02 \mathrm{~L}$, are shown in Figure 2 . It is clear that for the purely nonlocal case an increase in length scale parameter, $l_{c}$, makes the beam more flexible and consequently increases hte deflection of the beam. This is consistent with most of the reported results in the literature concerning Eringen nonlocal model (for instance Peddieson et al. (2003); Wang and Liew (2007); Reddy (2007); Wang et al. (2008)), whereas Di Paola et al. (2011) reported an opposite trend for constant $\xi_{1}$ and varying $l_{c}$. According to Figure 2 the transverse deflection of the beam can increase up to about $15 \%$ for $l_{c}=0.02 L$.

The results for the maximum normalized deflection for three different values of $l_{c}$ and varying phase parameter are shown in Figure 3 , where $\xi_{1}=0$ and $\xi_{1}=1$ 


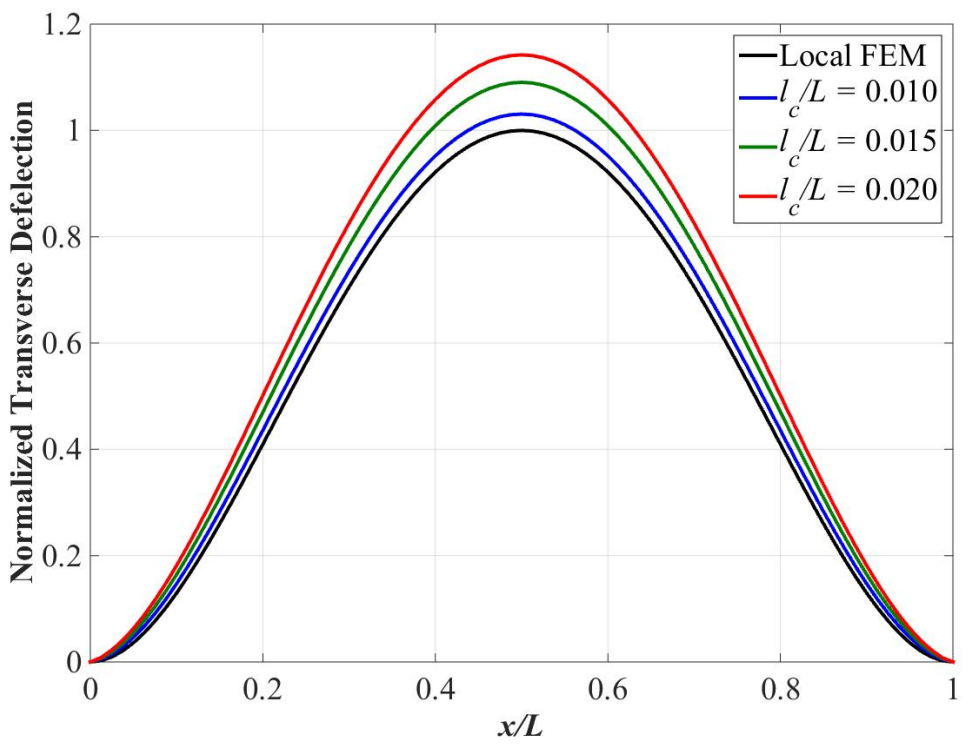

Figure 2: Normalized deflections of a clamped beam with uniformly distributed load.

correspond to purely nonlocal and purely local constitutive models, respectively. It is clear that the normalized deflection increases with a decrease in the phase parameter, that is, an increase in the nonlocal part of equation 27. One can conclude that for a clamped beam increase in $l_{c}$ and decrease in $\xi_{1}$ results in a more flexible beam. It is worth mentioning that higher $l_{c}$ and lower $\xi_{1}$ both denote higher nonlocal effects in the system.

In the local approach, the case of a homogeneous beam with constant cross section having symmetric boundary conditions and loading conditions can be further simplified with the use of the inherent symmetry of the model. Using the symmetry, one can model only half of the beam $[0, L / 2]$, and the displacement of the second half is the mirror image of the displacement of the first half. Due to the decrease in the domain to be modeled, the accuracy can be increased, i.e. the same number of elements in a smaller domain gives higher accuracy. Symmetry is present for a clamped beam with uniform distributed load. Use of symmetry in the nonlocal model of a clamped beam is examined next. The results for the case with symmetry are shown in Figure 4. Compared to the results shown in Figure 2, use of symmetry causes the beam to become more flexible. As stated by Pisano et al. (2009), the discretized Eringen nonlocal formulation will introduce some error near the boundaries of the model. This error is present in a zone with a dimension equal to the influence zone (which is the distance beyond which nonlocal 


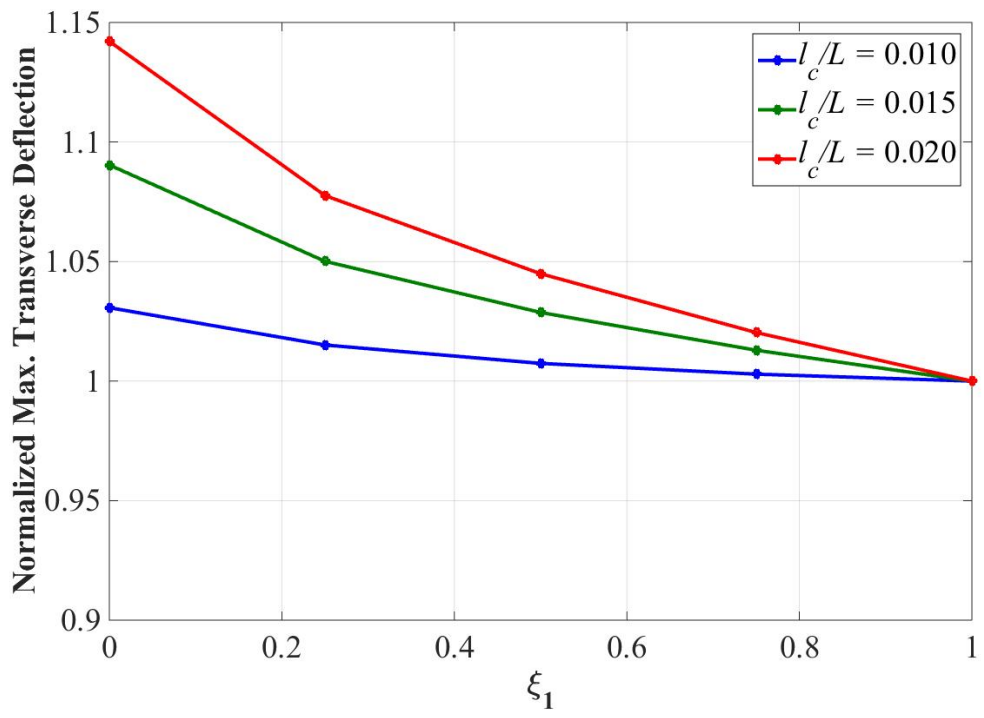

Figure 3: Normalized maximum deflections of a clamped beam with uniformly distributed load for different values of the length scale and phase parameters.

effects are neglected). This error is due to the fact that in the integral of equation 3 it is assumed that point $\mathbf{x}$ is embedded in an infinite medium. This assumption is violated at the boundaries of the medium and the integral will not result in unity. Therefore, as a result of the end conditions, the stiffness terms in this zone are approximated lower than the true value. This causes the noticeable difference in the deflection of the case with symmetry and without symmetry. It can be seen that the difference is more for higher values of $l_{c}$. This shows that reduction of the domain by use of symmetry is not allowable in nonlocal analysis, unless some conditions are enforced at the end conditions to modify the resulting error in the stiffness of these areas.

Note that the error caused by end conditions is present even in the case where the full beam is analyzed. However, since in the case of a full beam the boundary conditions at the ends of the beam involve a constraint on the transverse deflection, the reduction in stiffness will not affect the deflection to the extent of the case of a half beam. In the case of a half beam, the introduced boundary condition in the middle of the beam has a constraint on the slope of the beam and not the transverse deflection. As a result, the influence of the end conditions on the deflection will become more prominent with use of the symmetry. 


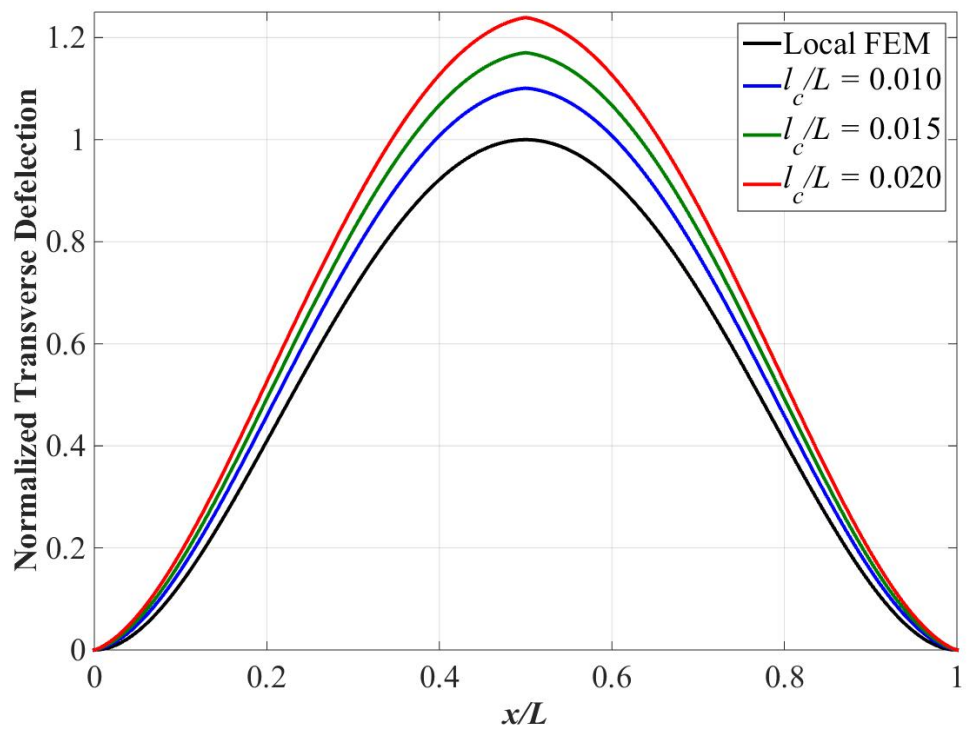

Figure 4: Normalized deflections of a clamped beam with uniformly distributed load (symmetry is used to model half of the beam).

\subsection{Simply Supported Beam with Uniformly Distributed Load}

Next the problem of a simply supported beam under uniformly distributed transverse load of intensity $q_{0}$ is studied. The maximum local deflection of this beam is $5 q_{0} l^{4} / 384 E I$. The normalized deflection of the beam for $\left(\xi_{1}=0\right)$ and 3 different values of $l_{c}$ is shown in Figure 5. The results shown in Figure 5 (simply supported beam) show a different trend as compared with those shown in Figure 2 (clamped beam). The deflection of the simply supported beam increases with the increase in the length scale parameter $l_{c}$. However, the increase is not very significant. Also note that the deflections derived from nonlocal theory are smaller than their local counterpart which is not in agreement with the softening effect reported in the literature.

Same as what was done for the clamped beam, the maximum normalized deflection for three different values of $l_{c}$ and varying phase parameter is shown in Figure 6. It is obvious that lowering the phase parameter makes the system more stiff; therefore, the tip deflection increases with increasing $\xi_{1}$. This is in contradicition with the results of the clamped beam (Figure 3). In general, for the simply supported beam decrease in $\xi_{1}$ decreases the deflection as opposed to other boundary conditions and the reported results in the literature. One should note that the kernel function used in this study is different from the one which gives 


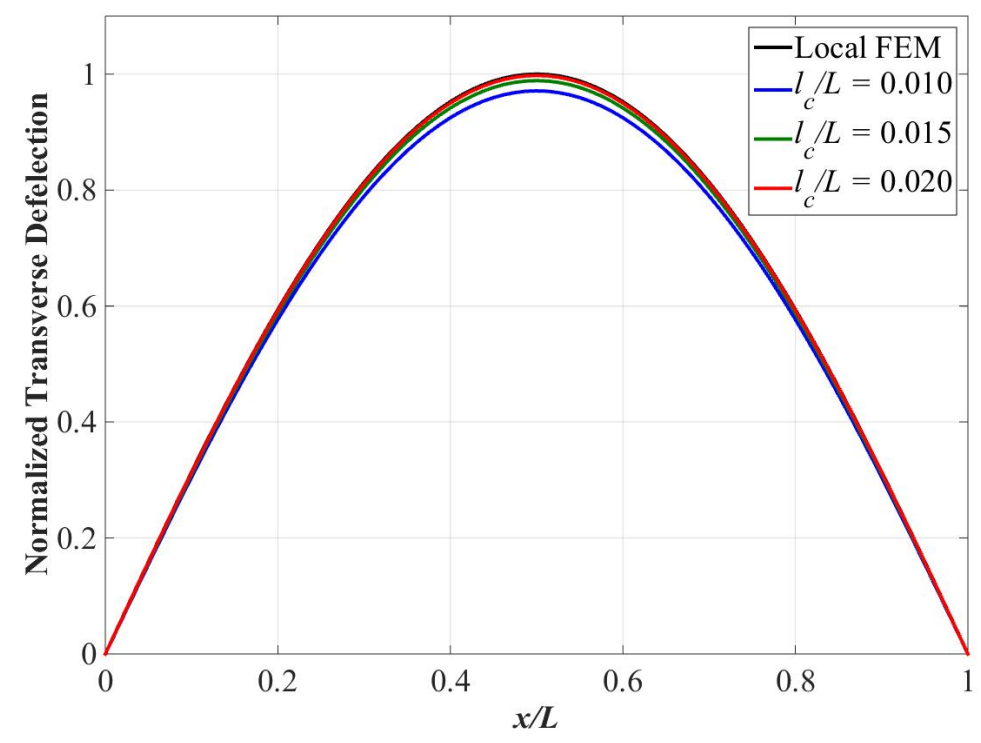

Figure 5: Normalized deflections of a simply supported beam with uniformly distributed load.

rise to Eringen's differential nonlocal model. The differential model comes from a kernel function with a Gaussian distribution; therefore, differences are to be expected. As will be shown next, the kernel function used here (equation 4) eliminates the paradox concerning the cantilever beam, however, it introduces another paradox for the simply supported beam.

Although for the case of a simply supported beam the nonlocal transverse deflections are smaller than the local deflections, by increasing the number of elements in the beam the results of the local and nonlocal analysis converge and the difference between the results will decrease. This is shown in Figure 7 for $l_{c}=0.01 L$ (for which according to Figure 5 the difference between local and nonlocal deflections was larger among the different values considered for $l_{c}$ ) and increasing number of elements.

Similar to the clamped beam, the analysis of the half beam with the use of ineherent symmetry of the simply supported beam was performed. It was once again concluded that huge errors will be introduced into the problem, if the stiffness of the end zone near the half beam is not modified.

\subsection{Cantilever Beam with Concentrated Load}

The third example deals with a cantilever beam with a point load at the free end. The normalized deflections are shown in Figure 8. The conventional (local) 


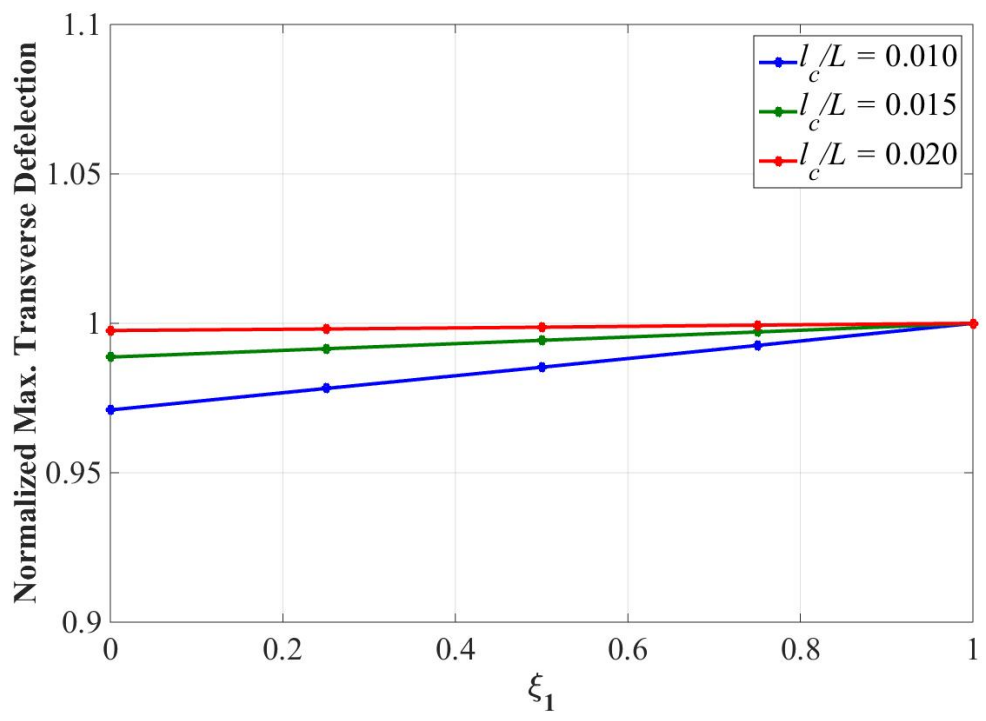

Figure 6: Normalized maximum deflections of a simply supported beam with uniformly distributed load for different values of the length scale and phase parameters.

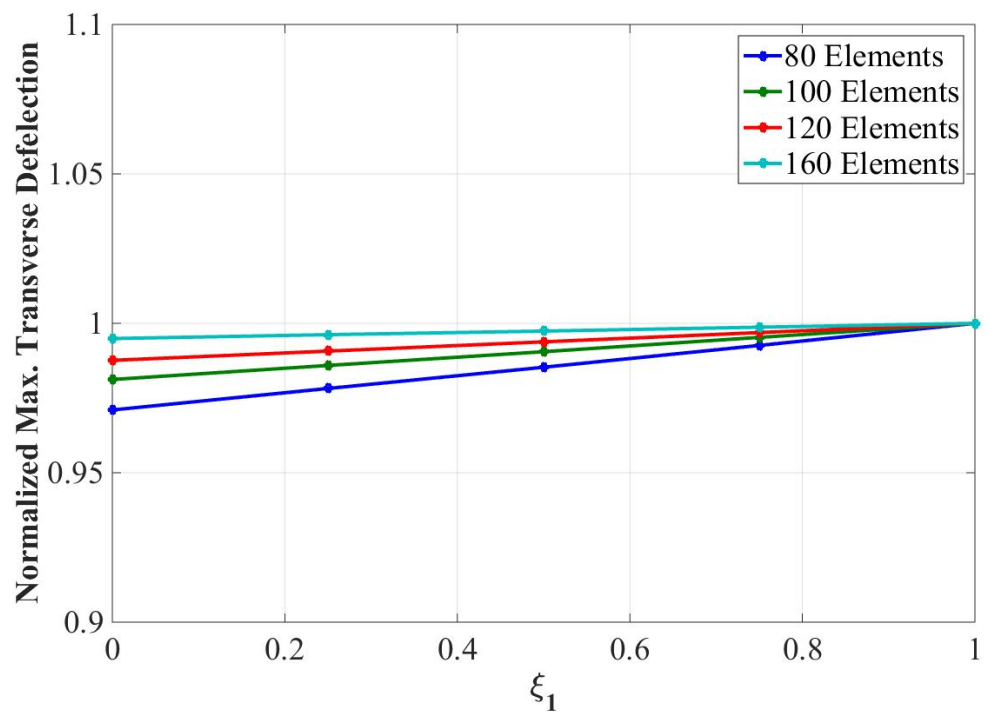

Figure 7: Normalized maximum deflections of a simply supported beam with uniform distributed load for different number of elements. 


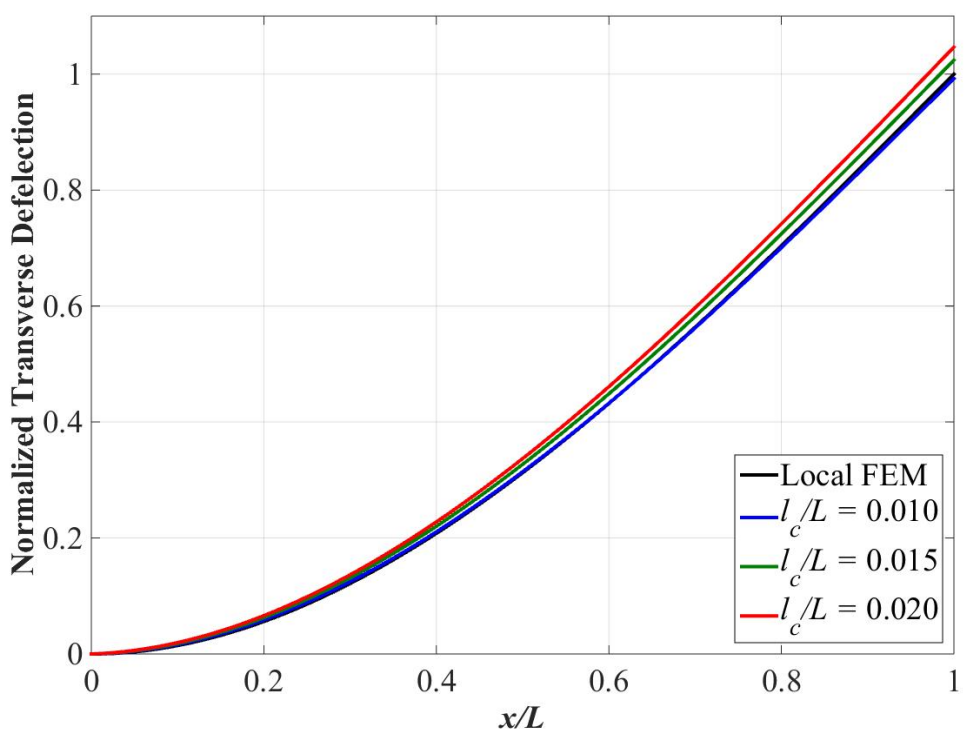

Figure 8: Results for a cantilever beam with point load at the free end, normalized by the local maximum deflection.

FEM result for the maximum deflection of a cantilever beam of length $L$ with constant $E I$ subjected to a concentrated load $P$ at the free end is $P L^{3} / 3 E I$. The deflections are normalized by this value to remove the effect of other parameters. The results shown in Figure 8 correspond to the value of $\xi_{1}=0$ which gives the original one-phase Eringen nonlocal formulation of equation 1 . The results show that as expected the increase in the length scale parameter $l_{c}$ makes the system more flexible and the deflections are increased which is in agreement with the trend of the clamped beam and the results shown in the literature (Peddieson et al., 2003; Wang and Liew, 2007; Reddy, 2007; Wang et al., 2008). Although an increase in the deflection is noticed, this increase is not very significant. For this case the maximum increase in the deflection of the tip of the beam is about $5 \%$ for $l_{c}=0.02 L$. The results for $l_{c}=0.01 L$ is almost the same as those of the conventional local FEM.

The normalized deflection of the tip of the beam for varying phase parameter $\xi_{1}$ is shown in Figure 9. The results are provided for three different values of $l_{c}$ and five values of $\xi_{1}$. Similar to the clamped beam, the results show that the deflections increase with the decrease in the phase parameter.

Overall the normalized tip deflection is generally greater than unity for varying $l_{c}$ and $\xi_{1}$. In Figure 9 the values of the normalized tip deflection for $l_{c}=0.01 L$ 


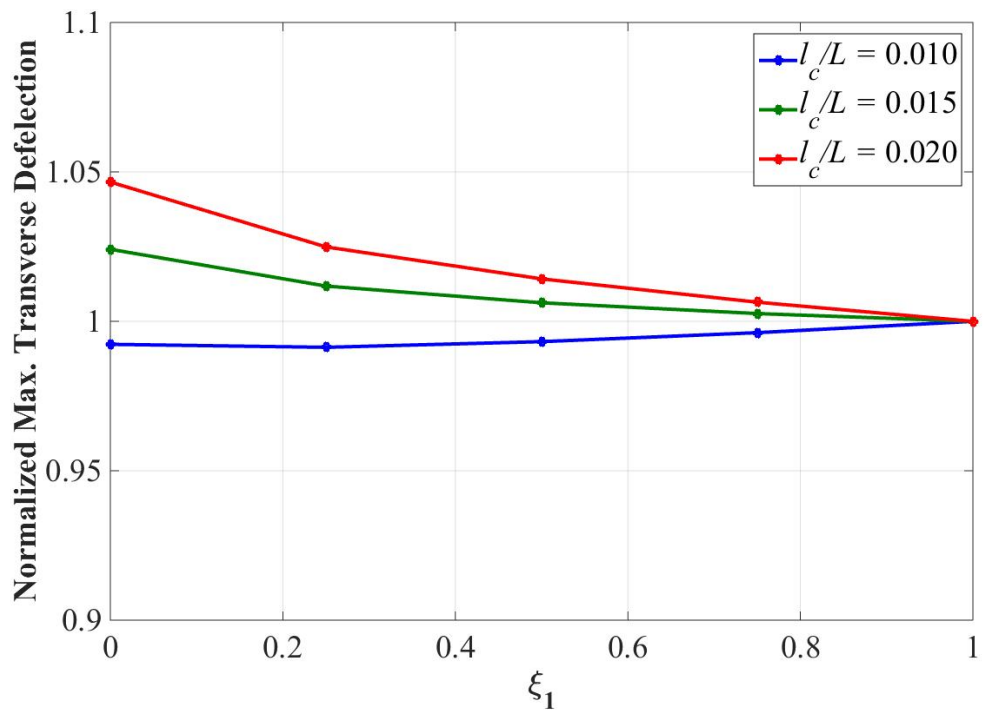

Figure 9: Normalized deflections of the tip of a cantilever beam with a point load at the tip for different values of length scale parameter and phase parameter.

and $\xi_{1}$ other than unity are slightly smaller than unity, the reason for which is discussed later. As opposed to the results reported in several studies (Peddieson et al., 2003; Wang and Liew, 2007; Wang et al., 2008; Challamel and Wang, 2008; Challamel et al., 2014) for the paradoxical case of a cantilever beam, it can be seen that the integral form of Eringen nonlocal model (with the present choice of kernel function) results in deflections which are different from its local counterpart.

\subsection{Cantilever Beam with Non-uniform Distributed Load}

Finally, the case of a cantilever beam with triangularly distributed load (Figure 1d) is studied here. The maximum deflection for the conventional case is $q_{0} L^{4} / 30 E I$. Normalized displacements for varying $l_{c}$ and $\xi_{1}=0$ are shown in Figure 10, and normalized deflections of the tip of the beam for different values of $l_{c}$ and $\xi_{1}$ are shown in Figure 11. The trend is the same as in the previous cases of a clamped beam and a cantilever beam with a point load. By comparing the plots of Figure 11 with those in Figure 9 one can realize that the increase in deflection due to nonlocal effects is more significant for the cantilever beam with the triangularly distributed load than the one with a point load at the tip. Although the results for a cantilever beam with uniformly distributed load is not brought here, the deflections of the case of the triangularly distributed loads even exceed those of the uniformly distributed load. This can be attributed to the derivations 


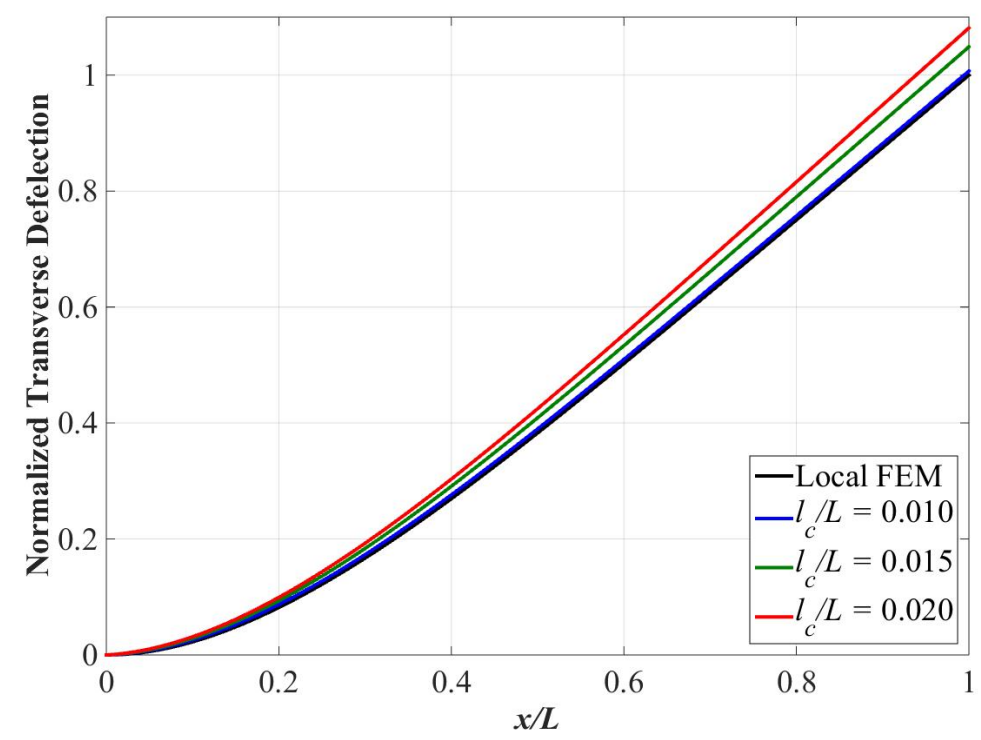

Figure 10: Normalized deflections of a cantilever beam with triangular distributed load.

by Reddy (2007) which shows that derivative of $q(x)$ (transverse distributed force) come into the picture in the differential form of the nonlocal Eringen model.

\section{Assessment of the Discrete Eringen Nonlocal Formulation}

As discussed in the introduction, most of the studies in the literature used the differential form of the Eringen nonlocal model. Now the discrete integral model is evaluated to determine its applicability to the problems.

After finite element discretization of the domain and development of elementwise equations, the elements are assembled using the connectivity matrix. The connectivity matrix is a rectangular array whose $i^{\text {th }}$ row gives the element numbers which are located in the influence zone of element $i$. A schematic view of the determination of the element numbers in the $i^{\text {th }}$ row of the connectivity matrix is shown in Figure 12. As shown in the figure, first the exponential term of equation 25 is constructed at the end nodes of the $i^{\text {th }}$ element (shown as a blue line). Next, the horizontal line corresponding to the criterion $e^{-l_{i} / l_{c}}$ is constructed (shown as a red line). The distance between the intersection of the red line and the blue line (from constructing the exponential term at end nodes of element $i$ ) is equal to $2 l_{i}+h_{i}$, where $l_{i}$ is the influence zone and $h_{i}$ is the length of element $i$, and it is named as the nonlocal zone of element $i$ (as shown in Figure 12). In the final step, 


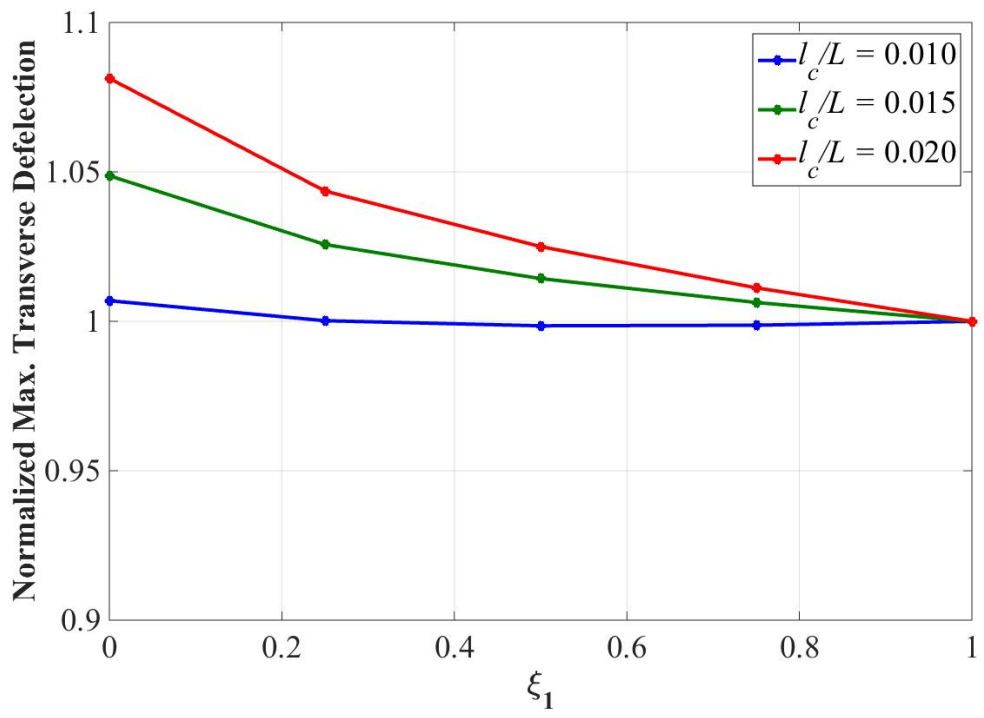

Figure 11: Normalized tip deflections of a cantilever beam with a triangular distributed load for different values of length scale and phase parameters.

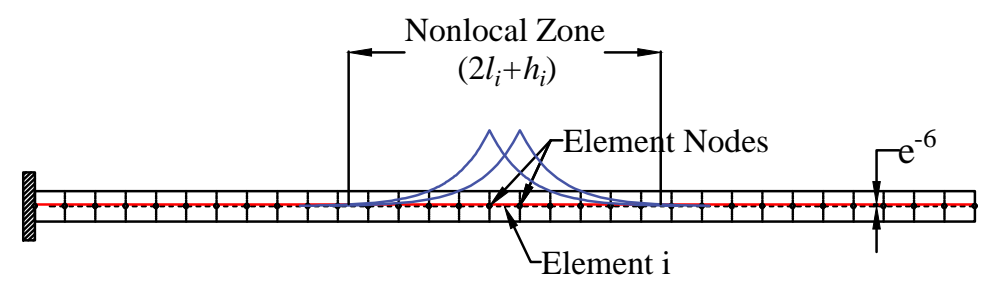

Figure 12: Determination of the elements falling in the influence zone of element $i$.

the elements remaining in the nonlocal zone of element $i$ are determined. These elements form the $i^{\text {th }}$ row of the connectivity matrix. This can be repeated for the rest of the elements to construct the connectivity matrix (Figure 13).

By examining Figure 13 one can see that for the elements located in a distance equal to the influence zone from the boundaries of the beam the integral equation (equation 3 ) is not satisfied. This causes the results to have some undesirable changes in these areas. On the other hand, as it is obvious from the figure, the nonlocal stiffness $\mathbf{k}_{e e^{\prime}}^{n l}$ depends highly on the mesh distribution ( $l_{c}$ is chosen to be much smaller than the length of the beam). The accuracy of the results in the integral form depends on the relative value of $l_{c}$ and the mesh size. If the mesh is very coarse, the finite element solution will not converge to the true solution. Therefore, to obtain accurate results the mesh size needs to be determined 


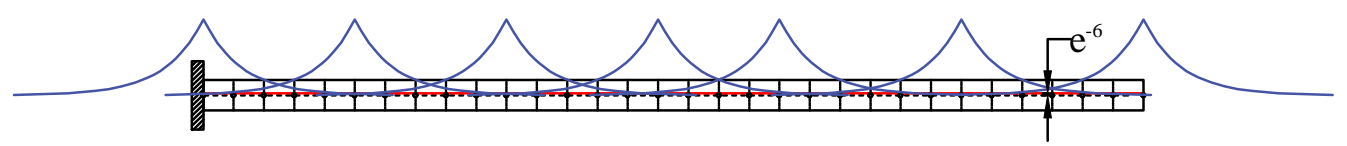

Figure 13: Constructing the connectivity matrix.

according to the length scale parameter, $l_{c}$. To illustrate this, the final results for the normalized maximum transverse deflection of a clamped beam with uniformly distributed load are shown in Table 1 for different values of length scale parameter, phase parameter, and number of elements. Note that the length of the beam is taken to be constant, therefore, by increasing the number of elements the mesh size is decreased. As can be seen, by increasing the number of elements the results start converging. For fewer number of elements, the maximum nonlocal deflection becomes less than the corresponding local response. However, the difference between the solutions obtained with meshes of 80, 90, and 100 elements becomes negligible (the figures provided in the previous sections all correspond to a mesh of 80 elements). This shows that if the integral form of the Eringen model is to be used, a sensitivity analysis should be conducted on the mesh size before final analysis. Note that according to the results presented in Table 1, the sensitivity of the solution to the number of elements decreases by increasing the value of the length scale parameter, $l_{c}$. This is due to the fact that increase in $l_{c}$ will result in the increase in the influence zone which will naturally increase the number of elements falling into the influence zone.

\section{Clamped vs. Simply Supported Beam}

Except for the simply supported beam, all of the numerical examples presented in section 3 show the following trends with the change in the nonlocal parameters, $l_{c}$ and $\xi_{1}$ :

1. The deflection increases with the increase in the length scale parameter, $l_{c}$.

2. The deflection increases with the decrease in the phase parameter, $\xi_{1}$.

3 . The nonlocal deflection $\left(\xi_{1} \neq 1\right)$ is generally larger than the local deflection.

For the simply supported beam the same trend with respect to variations of $l_{c}$ is noticed. However, the last two trends are not observed. The normalized deflection (nonlocal deflection divided by the maximum local deflection) is always less than or equal to unity (equality to 1 corresponds to the case of $\xi_{1}=0$ ); also, 
Table 1: Normalized maximum deflections for a clamped beam with uniformly distributed load.

\begin{tabular}{|c|c|c|c|c|c|c|}
\hline & \multirow{2}{*}{$\begin{array}{l}\text { Number of } \\
\text { elements }\end{array}$} & \multicolumn{5}{|c|}{ Normalized nonlocal deflection } \\
\hline & & $\xi_{1}=0.00$ & $\xi_{1}=0.25$ & $\xi_{1}=0.50$ & $\xi_{1}=0.75$ & $\xi_{1}=1.00$ \\
\hline & 20 & 0.6891 & 0.7469 & 0.8154 & 0.8982 & 1.0000 \\
\hline $\bar{\sigma}$ & 40 & 0.9252 & 0.9402 & 0.9579 & 0.9779 & 1.0000 \\
\hline$\|$ & 80 & 1.0307 & 1.0151 & 1.0074 & 1.0029 & 1.0000 \\
\hline 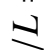 & 90 & 1.0387 & 1.0200 & 1.0106 & 1.0044 & 1.0000 \\
\hline$\sim$ & 100 & 1.0451 & 1.0239 & 1.0131 & 1.0056 & 1.0000 \\
\hline$n$ & 20 & 0.8638 & 0.8925 & 0.9246 & 0.9602 & 1.0000 \\
\hline$=$ & 40 & 1.0274 & 1.0124 & 1.0051 & 1.0014 & 1.0000 \\
\hline 11 & 80 & 1.0904 & 1.0501 & 1.0287 & 1.0129 & 1.0000 \\
\hline 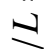 & 90 & 1.0960 & 1.0529 & 1.0304 & 1.0137 & 1.0000 \\
\hline 0 & 100 & 1.1003 & 1.0549 & 1.0317 & 1.0143 & 1.0000 \\
\hline ๑ & 20 & 0.9689 & 0.9717 & 0.9784 & 0.9880 & 1.0000 \\
\hline$\approx$ & 40 & 1.0951 & 1.0546 & 1.0310 & 1.0137 & 1.0000 \\
\hline & 80 & 1.1421 & 1.0776 & 1.0449 & 1.0203 & 1.0000 \\
\hline$\triangleq$ & 90 & 1.1466 & 1.0794 & 1.0460 & 1.0208 & 1.0000 \\
\hline- & 100 & 1.1495 & 1.0802 & 1.0464 & 1.0211 & 1.0000 \\
\hline
\end{tabular}

the normalized deflection decreases with decreasing $\xi_{1}$. To study this further, a simply supported beam with rotational springs at both ends, with equal spring constants of $\mu$, Figure 14, is considered. The case of $\mu=0$ corresponds to a simply supported beam, while $\mu \rightarrow \infty$ corresponds to a clamped beam. It is understood that one cannot reach infinity numerically, however, for a value of $\mu$ large enough the deflections of the clamped beam are attained.

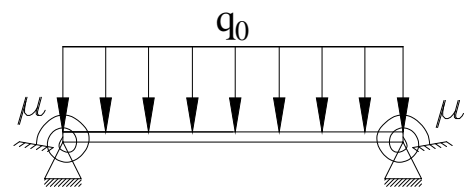

Figure 14: Simply supported beam with rotational spring with constant $\mu$ at both ends.

To see when the transition from the simply supported beam to the clamped beam occurs, maximum nonlocal deflection for different values of $\mu, l_{c}$, and $\xi_{1}$ is obtained. Once again the results are normalized with respect to the maximum local deflection. The results are tabulated in Table 2 for different values of the normalized rotational stiffness, $\mu L / E I$, normalized length scale parameter, $l_{c} / L$, 
and phase parameter $\xi_{1}$. The values of normalized deflection that are larger or equal to unity are highlighted in the table. For $\mu L / E I=e^{0}=1$, the results are almost the same as the simply supported beam (note that in this case, $\mu$ acquires a small value, $\mu=E I / L \neq 0$ ). The nonlocal simply supported beam behaves slightly stiffer compared to its local counterpart. Also note that the normalized deflection for $\mu L / E I=e^{10}$ and $\mu L / E I=e^{12}$ is the same and convergence to the clamped beam has been made. One can see that the nonlocal clamped beam is more flexible than the local clamped beam. As expected, the normalized deflection for $\xi_{1}=1$, purely local constitutive equation, will be exactly equal to 1 for all values of $l_{c} / L$ and $\mu L / E I$. For other values of $\xi_{1}$, with an increase in $l_{c}$ the change from a stiffer nonlocal system to a softer nonlocal system takes place with a lower value of the rotational stiffness spring $\mu$.

Finally, the normalized maximum transverse deflections for a wider range of values of $\xi_{1}$ and $\mu L / E I$ and a constant value of $l_{c} / L=0.02$ are shown in Figure 15 . Since $\mu$ changes from very small values to very large values, the $\mu L / E I$ is presented in the logarithmic form. The figure shows that for $l_{c} / l=0.02$, only a very small range of $\mu L / E I$ gives normalized values of less than unity. With the increase of $\mu$ the normalized deflection increases to about 1.14. Due to the difference in the kernel function used in this study as compared to the underlying kernel function for the differential Eringen nonlocal model, one should expect some differences to arise. The advantage of the integro-differential model with the exponential kernel function of equation 4 is that it solves the afore-mentioned controversy for cantilever beams which has many applications in science (Pereira, 2001; J. Pei and Thundat, 2004; Ekinci and Roukes, 2005; N. V. Lavrik and Datskos, 2004).

According to most of the studies in the literature Eringen nonlocal model has a softening effect (e.g. Peddieson et al. (2003); Wang et al. (2006); Wang and Liew (2007); Wang et al. (2008)), i.e. the displacements of the Eringen nonlocal model are typically higher than their local counterpart, while the frequencies are generally lower. However, they study by Reddy and El-Borgi (2014) and Li et al. (2015) showed that the behavior of the Eringen nonlocal model as compared to the corresponding local counterpart depends upon the type of loading and boundary conditions. Although the stiffness may increase in some cases, there exists some cases where it has a softening effect (Reddy and El-Borgi, 2014; Li et al., 2015). This further supports the results obtained in the present study. 
Table 2: Normalized maximum deflections of a simply supported beam with rotational springs at both ends for different values of $\mu L / E I, l_{c} / L, \xi_{1}$.

\begin{tabular}{|c|c|c|c|c|c|c|c|}
\hline & \multirow{2}{*}{$\mu L / E I$} & \multirow{2}{*}{$\begin{array}{l}\text { Max. Local } \\
\text { Deflection }\end{array}$} & \multicolumn{5}{|c|}{ Normalized Maximum Nonlocal Deflection } \\
\hline & & & $\xi_{1}=0.00$ & $\xi_{1}=0.25$ & $\xi_{1}=0.50$ & $\xi_{1}=0.75$ & $\xi_{1}=1.00$ \\
\hline \multirow{12}{*}{$\begin{array}{l}0 \\
0 \\
0 \\
11 \\
\stackrel{0}{\sim} \\
\sim\end{array}$} & $10^{0}$ & 1.3333 & 0.9710 & 0.9782 & 0.9853 & 0.9926 & 1.0000 \\
\hline & $10^{2}$ & 1.3325 & 0.9710 & 0.9781 & 0.9853 & 0.9926 & 1.0000 \\
\hline & $10^{4}$ & 1.2543 & 0.9728 & 0.9795 & 0.9862 & 0.9931 & 1.0000 \\
\hline & $2 \times 10^{4}$ & 1.1862 & 0.9745 & 0.9807 & 0.9870 & 0.9934 & 1.0000 \\
\hline & $5 \times 10^{0}$ & 1.0286 & 0.9785 & 0.9836 & 0.9889 & 0.9944 & 1.0000 \\
\hline & $10^{5}$ & 0.8593 & 0.9837 & 0.9872 & 0.9913 & 0.9955 & 1.0000 \\
\hline & $2 \times 10^{5}$ & 0.6769 & 0.9910 & 0.9919 & 0.9942 & 0.9970 & 1.0000 \\
\hline & $5 \times 10^{5}$ & 0.4800 & 1.0028 & 0.9992 & 0.9985 & 0.9989 & 1.0000 \\
\hline & $10^{6}$ & 0.3852 & 1.0118 & 1.0044 & 1.0015 & 1.0003 & 1.0000 \\
\hline & $10^{8}$ & 0.2680 & 1.0304 & 1.0149 & 1.0074 & 1.0028 & 1.0000 \\
\hline & $10^{10}$ & 0.2667 & 1.0307 & 1.0150 & 1.0074 & 1.0028 & 1.0000 \\
\hline & $10^{12}$ & 0.2667 & 1.0307 & 1.0150 & 1.0074 & 1.0028 & 1.0000 \\
\hline \multirow{12}{*}{$\begin{array}{l}n \\
0 \\
0 \\
11 \\
= \\
0 \\
-0\end{array}$} & $10^{0}$ & 1.3333 & 0.9887 & 0.9915 & 0.9943 & 0.9971 & 1.0000 \\
\hline & $10^{2}$ & 1.3325 & 0.9887 & 0.9915 & 0.9943 & 0.9971 & 1.0000 \\
\hline & $10^{4}$ & 1.2543 & 0.9897 & 0.9922 & 0.9947 & 0.9974 & 1.0000 \\
\hline & $2 \times 10^{4}$ & 1.1862 & 0.9907 & 0.9928 & 0.9952 & 0.9976 & 1.0000 \\
\hline & $5 \times 10^{0}$ & 1.0286 & 0.9939 & 0.9948 & 0.9964 & 0.9982 & 1.0000 \\
\hline & $10^{5}$ & 0.8593 & 0.9994 & 0.9983 & 0.9985 & 0.9991 & 1.0000 \\
\hline & $2 \times 10^{5}$ & 0.6769 & 1.0093 & 1.0041 & 1.0020 & 1.0007 & 1.0000 \\
\hline & $5 \times 10^{5}$ & 0.4800 & 1.0301 & 1.0160 & 1.0089 & 1.0039 & 1.0000 \\
\hline & $10^{6}$ & 0.3852 & 1.0483 & 1.0264 & 1.0150 & 1.0066 & 1.0000 \\
\hline & $10^{8}$ & 0.2680 & 1.0897 & 1.0497 & 1.0285 & 1.0128 & 1.0000 \\
\hline & $10^{10}$ & 0.2667 & 1.0904 & 1.0501 & 1.0287 & 1.0129 & 1.0000 \\
\hline & $10^{12}$ & 0.2667 & 1.0904 & 1.0501 & 1.0287 & 1.0129 & 1.0000 \\
\hline \multirow{12}{*}{ 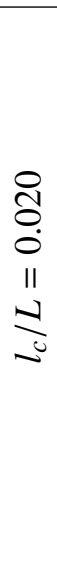 } & $10^{0}$ & 1.3333 & 0.9976 & 0.9981 & 0.9987 & 0.9994 & 1.0000 \\
\hline & $10^{2}$ & 1.3325 & 0.9975 & 0.9981 & 0.9987 & 0.9993 & 1.0000 \\
\hline & $10^{4}$ & 1.2543 & 0.9982 & 0.9986 & 0.9990 & 0.9995 & 1.0000 \\
\hline & $2 \times 10^{4}$ & 1.1862 & 0.9992 & 0.9990 & 0.9992 & 0.9996 & 1.0000 \\
\hline & $5 \times 10^{0}$ & 1.0286 & 1.0024 & 1.0009 & 1.0004 & 1.0001 & 1.0000 \\
\hline & $10^{5}$ & 0.8593 & 1.0092 & 1.0046 & 1.0026 & 1.0011 & 1.0000 \\
\hline & $2 \times 10^{5}$ & 0.6769 & 1.0225 & 1.0120 & 1.0068 & 1.0031 & 1.0000 \\
\hline & $5 \times 10^{5}$ & 0.4800 & 1.0523 & 1.0283 & 1.0163 & 1.0074 & 1.0000 \\
\hline & $10^{6}$ & 0.3852 & 1.0792 & 1.0431 & 1.0249 & 1.0112 & 1.0000 \\
\hline & $10^{8}$ & 0.2680 & 1.1410 & 1.0771 & 1.0446 & 1.0201 & 1.0000 \\
\hline & $10^{10}$ & 0.2667 & 1.1420 & 1.0776 & 1.0449 & 1.0203 & 1.0000 \\
\hline & $10^{12}$ & 0.2667 & 1.1420 & 1.0776 & 1.0449 & 1.0203 & 1.0000 \\
\hline
\end{tabular}




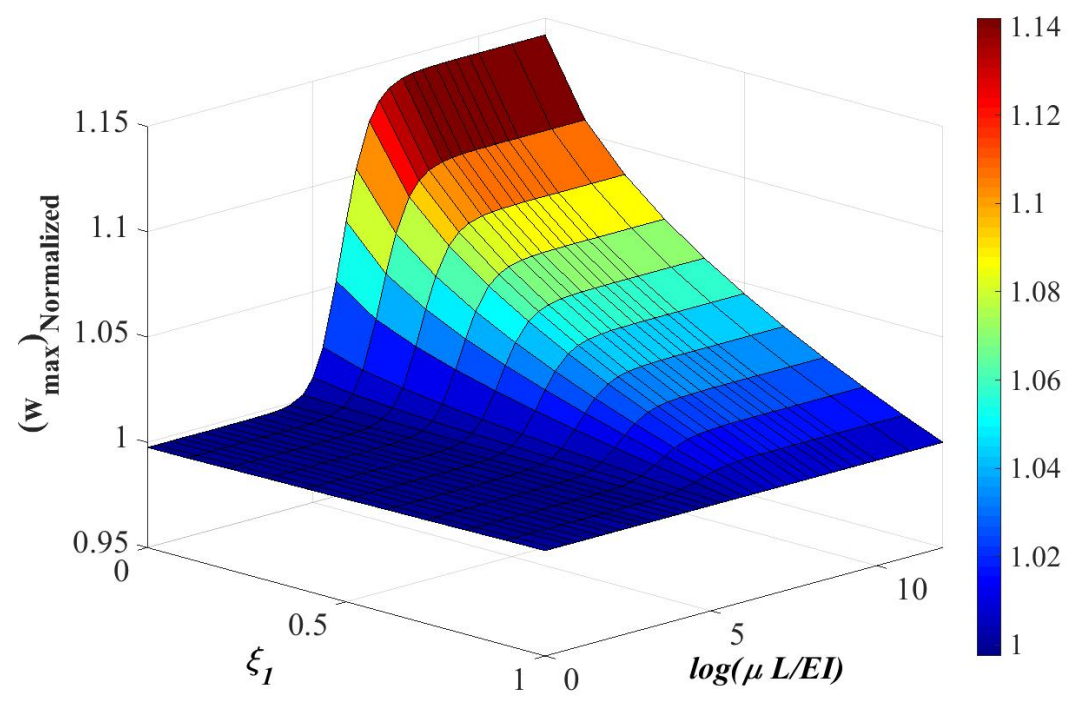

Figure 15: Normalized maximum deflections of a simply supported beam with rotational springs at both ends, $l_{c} / L=0.02$, varying value of $\xi_{1}$ and $\mu L / E I$.

\section{Conclusions}

A unified nonlocal integro-differential model is proposed as a generalization of Eringen's nonlocal model. In the present study the constitutive equation is written as a linear combination of local and nonlocal constitutive theories. The present model gives rise to two key parameters, namely the length scale parameter, $l_{c}$, and the phase parameter, $\xi_{1}$. General three-dimensional finite element formulation for the integro-differential model is presented. Then the results are specialized to the case of the Euler-Bernoulli beam theory. It is shown through numerical examples that the nonlocal beam is more flexible than its local counterpart for most boundary conditions (other than the case of a simply supported beam). The paradox surrounding the cantilever beam disappears with the integro-differential model proposed herein, which is very beneficial in the study of micro- and nanocantilevers. This can also be attributed to the different choice of kernel function as compared to the underlying kernel function for the differential form of Eringen's nonlocal model.

As shown in this study, the accuracy of the discrete form of the integral model depends on the mesh size, and it converges to the true solution by increasing the number of elements. However, note that due to the existence of nonlocal stiffness matrix, $\mathbf{k}_{n l}^{e e^{\prime}}$, increasing the number of nonlocal interactions will result 
in a global stiffness matrix with more nonzero terms (i.e., larger bandwidth) and consequently higher memory demand. The determination of the elements which are within the influence zone of a specific element becomes more challenging for two-dimensional and three-dimensional problems, which is a topic of future study. These are most likely the reasons why most of the previous studies made use of the differential form of the Eringen model. However, the integral form can be used to handle the problematic case of cantilever beams.

Application of the integro-differential model to other beam theories (e.g., the Timoshenko and Reddy third-order beam theories) is straightforward. However, extension and application of the ideas presented herein to plates and shells is more challenging and requires attention. It is also interesting to see how the integrodifferential model predicts the vibration response in fundamental and higher modes of vibration. It is hoped that with the publication of the present paper, a number of other studies will ensue.

\section{References}

\section{References}

Arash, B., Wang, Q., 2012. A review on the application of nonlocal elastic models in modeling of carbon nanotubes and graphenes. Computational Materials Science 51 (1), $303-313$.

Challamel, N., Wang, C. M., 2008. The small length scale effect for a non-local cantilever beam: a paradox solved. Nanotechnology 19 (34), 345703(7).

Challamel, N., Zhang, Z., Wang, C. M., Reddy, J. N., Wang, Q., Michelitsch, T., Collet, B., 2014. On nonconservativeness of Eringen's nonlocal elasticity in beam mechanics: Correction from a discrete-based approach. Archive of Applied Mechanics 84 (9-11), 1275-1292.

Di Paola, M., Failla, G., Sofi, A., Zingales, M., 2011. A mechanically based approach to non-local beam theories. International Journal of Mechanical Sciences $53(9), 676-687$.

Ekinci, K. L., Roukes, M., 2005. Nanoelectromechanical systems. Review of Scientific Instruments 76 (6), 061101(12).

Eringen, A. C., 1972a. Linear theory of nonlocal elasticity and dispersion of plane waves. International Journal of Engineering Science 10 (5), 425-435. 
Eringen, A. C., 1972b. Nonlocal polar elastic continua. International Journal of Engineering Science 10 (1), 1-16.

Eringen, A. C., 1983. On differential equations of nonlocal elasticity and solutions of screw dislocation and surface waves. Journal of Applied Physics 54 (9), 4703-4710.

Eringen, A. C., 1987. Theory of nonlocal elasticity and some applications. Res mechanica 21 (4), 313-342.

Eringen, A. C., 2002. Nonlocal Continuum Field Theories. Springer, New York, NY.

Eringen, A. C., Edelen, D. G. B., 1972. On nonlocal elasticity. International Journal of Engineering Science 10 (3), 233-248.

J. Pei, F. Tian, F., Thundat, T., 2004. Glucose biosensor based on the microcantilever. Analytical Chemistry 76 (2), 292-297.

Kröner, E., 1967. Elasticity theory of materials with long range cohesive forces. International Journal of Solids and Structures 3 (5), 731-742.

Krumhansl, J., 1968. Some considerations of the relation between solid state physics and generalized continuum mechanics. In: Kröner, E. (Ed.), Mechanics of Generalized Continua. IUTAM Symposia. Springer Berlin Heidelberg, pp. 298-311.

Kunin, I. A., 1968. The theory of elastic media with microstructure and the theory of dislocations. In: Kröner, E. (Ed.), Mechanics of Generalized Continua. IUTAM Symposia. Springer Berlin Heidelberg, pp. 321-329.

Li, C., Yao, L., Chen, W., Li, S., 2015. Comments on nonlocal effects in nanocantilever beams. International Journal of Engineering Science 87 (0), 47 - 57.

Lu, P., Lee, H. P., Lu, C., Zhang, P. Q., 2006. Dynamic properties of flexural beams using a nonlocal elasticity model. Journal of Applied Physics 99 (7), 073510(9).

N. V. Lavrik, M. J. S., Datskos, P. G., 2004. Cantilever transducers as a platform for chemical and biological sensors. Review of Scientific Instruments 75 (7), 2229-2253. 
Peddieson, J., Buchanan, G. R., McNitt, R. P., 2003. Application of nonlocal continuum models to nanotechnology. International Journal of Engineering Science 41 (3-5), 305-312.

Pereira, R. S., 2001. Atomic force microscopy as a novel pharmacological tool. Biochemical Pharmacology 62 (8), 975 - 983.

Pisano, A. A., Fuschi, P., 2003. Closed form solution for a nonlocal elastic bar in tension. International Journal of Solids and Structures 40 (1), 13-23.

Pisano, A. A., Sofi, A., Fuschi, P., 2009. Nonlocal integral elasticity: 2D finite element based solutions. International Journal of Solids and Structures 46 (21), 3836-3849.

Polizzotto, C., 2001. Nonlocal elasticity and related variational principles. International Journal of Solids and Structures 38 (42-43), 7359-7380.

Rahmani, O., Pedram, O., 2014. Analysis and modeling the size effect on vibration of functionally graded nanobeams based on nonlocal timoshenko beam theory. International Journal of Engineering Science 77 (0), 55 - 70.

Reddy, J. N., 2002. Energy Principles and Variational Methods in Applied Mechanics, Second Edition (the Third Edition to appear in 2016). John Wiley \& Sons, New York, NY.

Reddy, J. N., 2006. An Introduction to the Finite Element Method. McGraw Hill, New York, NY.

Reddy, J. N., 2007. Nonlocal theories for bending, buckling and vibration of beams. International Journal of Engineering Science 45 (2-8), 288-307.

Reddy, J. N., 2010. Nonlocal nonlinear formulations for bending of classical and shear deformation theories of beams and plates. International Journal of Engineering Science 48 (11), 1507 - 1518.

Reddy, J. N., 2013. An Introduction to Continuum Mechanics, Second Edition. Cambridge University Press, New York, NY.

Reddy, J. N., El-Borgi, S., 2014. Eringen's nonlocal theories of beams accounting for moderate rotations. International Journal of Engineering Science 82 (0), 159 -177 . 
Reddy, J. N., El-Borgi, S., Romanoff, J., 2014. Non-linear analysis of functionally graded microbeams using Eringen's non-local differential model. International Journal of Non-Linear Mechanics 67 (0), 308 - 318.

Salehipour, H., Shahidi, A., Nahvi, H., 2015. Modified nonlocal elasticity theory for functionally graded materials. International Journal of Engineering Science $90(0), 44-57$.

Shakouri, A., Lin, R. M., Ng, T. Y., 2009. Free flexural vibration studies of doublewalled carbon nanotubes with different boundary conditions and modeled as nonlocal euler beams via the Galerkin method. Journal of Applied Physics 106 (9), 094307(10).

Silling, S. A., 2000. Reformulation of elasticity theory for discontinuities and long-range forces. Journal of the Mechanics and Physics of Solids 48 (1), 175209.

Sudak, L. J., 2003. Column buckling of multiwalled carbon nanotubes using nonlocal continuum mechanics. Journal of Applied Physics 94 (11), 7281-7287.

Thai, H.-T., 2012. A nonlocal beam theory for bending, buckling, and vibration of nanobeams. International Journal of Engineering Science 52 (0), 56 - 64.

Thai, H.-T., Vo, T. P., 2012. A nonlocal sinusoidal shear deformation beam theory with application to bending, buckling, and vibration of nanobeams. International Journal of Engineering Science 54 (0), 58 - 66.

Wang, C. M., Kitipornchai, S., Lim, C. W., Eisenberger, M., 2008. Beam bending solutions based on nonlocal Timoshenko beam theory. Journal of Engineering Mechanics 134 (6), 475-481.

Wang, C. M., Zhang, Y. Y., Ramesh, S. S., Kitipornchai, S., 2006. Buckling analysis of micro- and nano-rods/tubes based on nonlocal Timoshenko beam theory. Journal of Physics D: Applied Physics 39 (17), 3904-3909.

Wang, Q., Liew, K. M., 2007. Application of nonlocal continuum mechanics to static analysis of micro- and nano-structures. Physics Letters A 363 (3), 236242.

Wang, Q., Wang, C. M., 2007. The constitutive relation and small scale parameter of nonlocal continuum mechanics for modelling carbon nanotubes. Nanotechnology 18 (7), 075702(4). 